\title{
Frontières
}

\section{Les liaisons sémantiques existant entre les catégories " naissance/jeunesse/vie " et " maturité/vieillesse/mort "}

\section{Jean-Michel Vidal}

Volume 18, numéro 1, automne 2005

Hélas, célébrer la mort

URI : https://id.erudit.org/iderudit/1074314ar

DOI : https://doi.org/10.7202/1074314ar

Aller au sommaire du numéro

Éditeur(s)

Université du Québec à Montréal

ISSN

1180-3479 (imprimé)

1916-0976 (numérique)

Découvrir la revue

Citer cet article

Vidal, J.-M. (2005). Les liaisons sémantiques existant entre les catégories « naissance/jeunesse/vie » et « maturité/vieillesse/mort ». Frontières, 18(1), 45-50. https://doi.org/10.7202/1074314ar
Résumé de l'article

Dans un premier temps et à l'aide d'exemples pris en Occident et en Afrique de l'Ouest (principalement chez les Dogons du Mali), cet article se propose d'explorer à travers les « logiques » des cycles de vie, certaines caractéristiques relatives aux concepts de naissance, de jeunesse, de vieillesse et de mort. Les liens qui se tissent entre ces différents concepts et leurs représentations s'agencent et s'entremêlent différemment au fil des cultures et du temps pour nous permettre, entre autres, d'affronter le chaos existentiel que nous expérimentons tous lorsque " jeunesse » et « mort » se juxtaposent en se télescopant comme c'est le cas lors de la mort d'un enfant. Dans un deuxième temps, cet article s'attache alors à comprendre les glissements sémantiques de ces catégorisations qui oscillent entre un ordre purement biologique et un ordre essentiellement symbolique. Ces glissements sémantiques sont à lire et à comprendre sur leurs horizons macrocontextuel, microcontextuel et individuel, mais aussi en fonction des normes et des valeurs culturelles et temporelles qui les sous-tendent et les rationalisent. 
Résumé

Dans un premier temps et à l'aide d'exemples pris en Occident et en Afrique de l'Ouest (principalement chez les Dogons du Mali), cet article se propose d'explorer à travers les "logiques» des cycles de vie, certaines caractéristiques relatives aux concepts de naissance, de jeunesse, de vieillesse et de mort. Les liens qui se tissent entre ces différents concepts et leurs représentations s'agencent et s'entremêlent différemment au fil des cultures et du temps pour nous permettre, entre autres, d'affronter le chaos existentiel que nous expérimentons tous lorsque "jeunesse» et "mort» se juxtaposent en se télescopant comme c'est le cas lors de la mort d'un enfant. Dans un deuxième temps, cet article s'attache alors à comprendre les glissements sémantiques de ces catégorisations qui oscillent entre un ordre purement biologique et un ordre essentiellement symbolique. Ces glissements sémantiques sont à lire et à comprendre sur leurs horizons macrocontextuel, microcontextuel et individuel, mais aussi en fonction des normes et des valeurs culturelles et temporelles qui les sous-tendent et les rationalisent.

Mots clés: sémantique - naissance jeunesse - vieillissement - mort Dogons du Mali.

\section{Abstract}

First, using examples from Western and West-African (especially the Dogon's tribe from Mali) cultures, this article explores the caracteristics of concepts like birth, youth, aging and death. Through history and time, in every culture, these different concepts and representations develop ties. They help us face the existential chaos we feel when "youth" meets "death", as is the case when a child dies. Secondly, this article tries to understand the different semantic meanings of these concepts, going from biology to symbolism. The transformation in the definition of these concepts needs to be understood in the context (values and laws) in which they occured and in which they make sense.

Keywords: semantic - birth - youth aging - death - Mali's Dogon.

\section{Les liaisons sémantiques existant entre les catégories "naissance/jeunesse/vie» et "maturité/vieillesse/mort»}

\section{Jean-Michel Vidal, M.D., Ph.D., ${ }^{1}$ Université de Montréal.}

Comment faire le deuil d'un être qui n'a pas pu vieillir, mûrir en quelque sorte et donc qui n'a pu connaître la vie? Comment surmonter la révolte que la disparition d'un enfant, voire de son propre enfant, suscite? Comment affronter cette cruelle aberration de l'ordre divin ou naturel qui fait nous précéder dans la mort celui-là même qui était censé nous survivre et nous perpétuer?

Ces questions tous les peuples de la Terre se les posent. Chacun à sa façon tentera d'apporter des réponses collectives ou individuelles. En ce domaine, il n'y aura pas de réponse toute faite. Au mieux, comme je me propose de le faire, il est possible d'esquisser à grands traits certaines caractéristiques culturelles et psychosociales entourant la mort d'un enfant. Il faut savoir cependant que ces caractéristiques sont toujours à lire sur leurs horizons macrocontextuel, microcontextuel et individuel. De plus, les « règles » culturelles que nous nous donnons collectivement sont toujours de nature temporelle et donc soumises à transformation. Elles existent, pourrait-on dire, pour qu'individuellement nous puissions les manipuler, les transformer, les adapter et parfois même les transgresser. On parlera alors de l'arbitraire du culturel.

Afin de rendre compte de ces « réalités» subjectives entourant la mort d'un enfant, je me propose dans un premier temps de faire un état des lieux en commençant par ce que l'on observe en Occident puis de les mettre en perspective avec d'autres réalités princi- palement issues d'Afrique de l'Ouest et des Dogons du Mali. Cette approche relativiste et donc culturaliste de la naissance et de la mort mais aussi des concepts relatifs à la jeunesse et la vieillesse qui leur sont intimement reliés me permettra de dégager les «logiques» et les constructions culturelles mises en place par les individus afin de faire face au chaos qu'irrémédiablement la mort de l'enfant provoque.

Dans un deuxième temps, à l'aide des catégorisations conceptuelles et sémantiques dégagées de part et d'autre, à la fois en Occident et en Afrique de l'Ouest chez les Dogons, je m'intéresserai à comprendre les glissements sémantiques qui se sont opérés en Occident entre: 1) les réalités biologiques de ces concepts (naissance/jeunesse et vie reliées à leurs opposants naturels: maturité/ vieillesse et mort) et 2) le nouvel ordre symbolique de ces réalités dans le monde occidental biomédical d'aujourd'hui où naissance/jeunesse et mort sont désormais reliées à leurs nouveaux antagonistes maturité/vieillesse et vie.

\section{NAISSANCE/JEUNESSE/VIE. S'AGIT-IL LÀ D'UNE DISPOSITION PARTICULIÈREMENT RATIONNELLE? DANS LES SOCIÉTÉS INDUSTRIELLES OCCIDENTALES: EXEMPLES PRIS EN EUROPE \\ ET AU QUÉBEC \\ En Occident, et principalement pour les fins de cet article en Amérique du Nord et en Europe, si l'on se réfère aux nom- breuses publications qu'ont suscitées ces thématiques relatives à la mort (Thomas,}


2000, 2003; Des Aulniers, 1996; Raimbault, 1996), la mort de l'enfant est toujours abordée, étudiée d'une façon spécifique. Nous ne réagissons pas de la même manière face à la mort d'un enfant et face à la mort d'un adulte. Les enfants dans la tradition judéo-chrétienne et selon les principes philosophico-religieux qui sous-tendent celle-ci, sont particulièrement reliés à l'innocence, à la pureté et à l'angélisme. Toutes choses qui font des enfants des êtres humains à part entière qui s'inscrivent dans un espace virginal les tenant éloignés de la culpabilité, du péché et de la mort. En ce qui concerne la mort de l'enfant, Ginette Raimbault pédo-psychologue et psychanalyste (1996) remarque qu'il est important de s'opposer d'emblée à une idée «reçue», à un a priori qui part du principe que la mort d'un être cher a pour effet premier de rassembler et d'unir les membres d'une même famille. Cette conception qui parfois peut être vraie lorsqu'il s'agit de la perte d'un parent plus âgé nécessite cependant d'être nuancée lorsque c'est un enfant qui décède. Car ce qui se joue alors, dans la famille de cet enfant, est de l'ordre d'un désordre structurel familial profond, se situant précisément très loin de cette idée $\mathrm{d}^{\text {' } ~}$ unicité », de front commun rassembleur de tous les membres d'une même famille.

Si l'aléatoire de la mort est déjà en soi une chose difficilement acceptable, celle-ci l'est d'autant plus lorsque c'est un enfant qui disparaît. En effet, il est aberrant, absurde, illogique que nos enfants nous précèdent dans la mort. Cet état de fait vient créer une rupture de l'ordre établi, une cassure structurelle qui instaure un certain chaos. Ainsi, à l'intérieur même de la structure familiale occidentale, dite nucléaire, loin d'unir la famille, la mort d'un enfant la met souvent en crise. Dans nos sociétés occidentales, de façon caricaturale et à des degrés divers, les professionnels de la santé, les aidants naturels, tous à leur façon font état de ce chaos familial qui suit la mort d'un enfant. Une œuvre cinématographique italienne $L a$ Stanza Del Figlio (Nanni Morretti, 2002) se penche sur cette situation de chaos et se charge de mettre en scène ce qui s'installe dans une famille lorsqu'un enfant décède. Le film montre que même psychanalyste (donc plus accoutumé à entendre la souffrance d'autrui), le père ne fait pas mieux que n'importe quel père dans une telle situation. Il ne parvient pas à tenir le chaos à distance: il s'enferme dans sa douleur, cède aux remords et à la culpabilité, s'éloigne de sa femme et de son autre enfant et met ce qui reste de la famille en péril de désunion. Ce père se reprochera ainsi d'avoir plutôt investi dans son travail au détriment de son enfant. La mère, elle aussi accablée par le chagrin, refusera sa couche à son mari. Et enfin, pour l'enfant restant, à la culpabilité de survivre se rajoutera désormais le poids d'être seule face à sa douleur mais surtout face à celle de ses parents. L'expérience du chaos généré par cette «insupportable vacuité de l'affliction et du remords» qui accompagne la mort d'un enfant, peut durer longtemps et s'étendre de proche en proche aux autres membres d'une même famille et parfois s'attaquer à la structure familiale elle-même au risque de la détruire.

Les impacts de ce chaos sont nombreux et ils dépendent, je l'ai indiqué, à la fois de l'histoire familiale, de la genèse de celle-ci mais aussi de l'histoire subjective de chacun (c'est-à-dire en fait de l'histoire de la famille avant la perte de l'enfant, des deuils précédents et du facteur subjectif personnel propre à chacun).

\section{LOIN D'UNIR LA FAMILLE, LA MORT D'UN ENFANT \\ LA MET SOUVENT EN CRISE.}

Ainsi selon Raimbault (1996), les impacts de ce chaos peuvent se traduire dans nos comportements de façon très différente. Les comportements «compensateurs » ou «réparateurs» peuvent prendre diverses formes: désir d'enfantement, militantisme, l'entrée en religion (ou réactivation du religieux), spiritisme, ou à plus long terme, des comportements qui entraînent des ruptures conjugales (G. Raimbault donne alors l'exemple de Mahler, de Mallarmé), ou encore différents modes d'évasion (alcool, drogue) ou l'apparition de troubles psychiques (visions, hallucinations) ou plus heureux (mais pour qui?), le développement d'une créativité ravivée, exacerbée, d'un véritable exutoire artistique. Raimbault cite l'exemple de Victor Hugo à la mort de sa fille Léopoldine ainsi que celui (plus proche de nous) d'Éric Clapton à la mort de son fils.

Ce chaos se «compense» donc de façons bien différentes les unes des autres. Pourtant ces différentes réactions mettent toutes en cause, à un titre ou l'autre, l'ordre relationnel de la personne et son rapport à l'autre. Comme si en Occident ce chaos venait révéler en creux,un déficit dans la sociabilité des individus. Une zone non investie se situant à l'intersection du personnel et du familial, de l'intime et du partagé, des limites mais aussi des failles de nos modes de prise en charge collective et de l'individualisme qui nous caractérise tant. Tout se passe un peu comme si ce chaos causé par la mort et la disparition d'un enfant contribuait à plonger l'individu dans un espace intermédiaire transitionnel qui, dans nos cultures occidentales, devient de moins en moins balisé, voire qui n'est plus balisé du tout, en raison entre autres du nivellement du symbolique, principalement amené par une perte de l'idée d'une transcendance au profit d'un monde matériel, objectif, cartésien, et donc soumis aux lois de la physique, de la chimie, de la biologie et du rationnel.

En règle générale, la mort est intimement reliée à l'inconnu, à l'irrationnel et plus encore à l'incapacité d'admettre que nous puissions être brutalement séparés d'êtres qui nous étaient les plus proches. La mort de l'enfant vient justement nous parler avec encore plus d'acuité de cet irrationnel et de l'extrême difficulté que nous avons alors à donner du sens au chaos dans lequel nous nous trouvons plongés. Cette quête de sens qui nous anime devant tout évènement irrationnel nous est pourtant essentielle pour vivre, et pour survivre à nos enfants.

Sans vouloir adopter une approche anthropologique de type fonctionnaliste, qui voit dans la culture un système ayant pour rôle essentiel de nous permettre d'appréhender le réel et de donner du sens à ce qui à priori n'en aurait pas, je m'inscris plutôt dans la lignée structuraliste de C. Lévi-Strauss lorsqu'il écrit: "Dire que la culture est fonctionnelle c'est une évidence, mais dire que tout fonctionne dans une culture est une absurdité» (Lévi-Strauss, 1997). En effet, il existe toujours une part d'inconnu, de «creux» de sens, de vacuité « existentielle» à laquelle nous devons tous faire face, et cela, dans toutes les cultures. Et la mort d'un enfant vient justement, dramatiquement même, révéler de façon toujours particulière cet espace vide de sens, d'où le chaos toujours relié à l'incohérence, l'incongruité, l'illogisme, l'inconvenance de la mort de l'enfant. Chaque culture tentera alors à sa façon d'essayer d'organiser ce chaos afin justement de lui donner un sens.

On vient d'évoquer que certains mécanismes parfois traumatiques peuvent s'enclencher et se révéler à la mort d'un enfant, car cette dernière s'inscrit en faux dans l'ordre des choses et va à l'encontre de ce qui est considéré comme «naturel» en Occident.

\section{DANS LES SOCIÉTÉS \\ TRADITIONNELLES AFRICAINES: EXEMPLE PRIS CHEZ LES DOGONS \\ DU MALI}

L'anthropologie des âges de la vie, développée par des auteurs comme Jacqueline Rabin (1966), Leila Pissani (1983) et Barbara Fiore $(1988,1990)$ de l'école italienne d'ethnologie, Anne-Marie Peatrik (2003), Françoise Héritier-Augé (19911993), Paul Riesman (1992) pour n'en citer que quelques-uns, est un courant en anthropologie socioculturelle qui s'inté- 


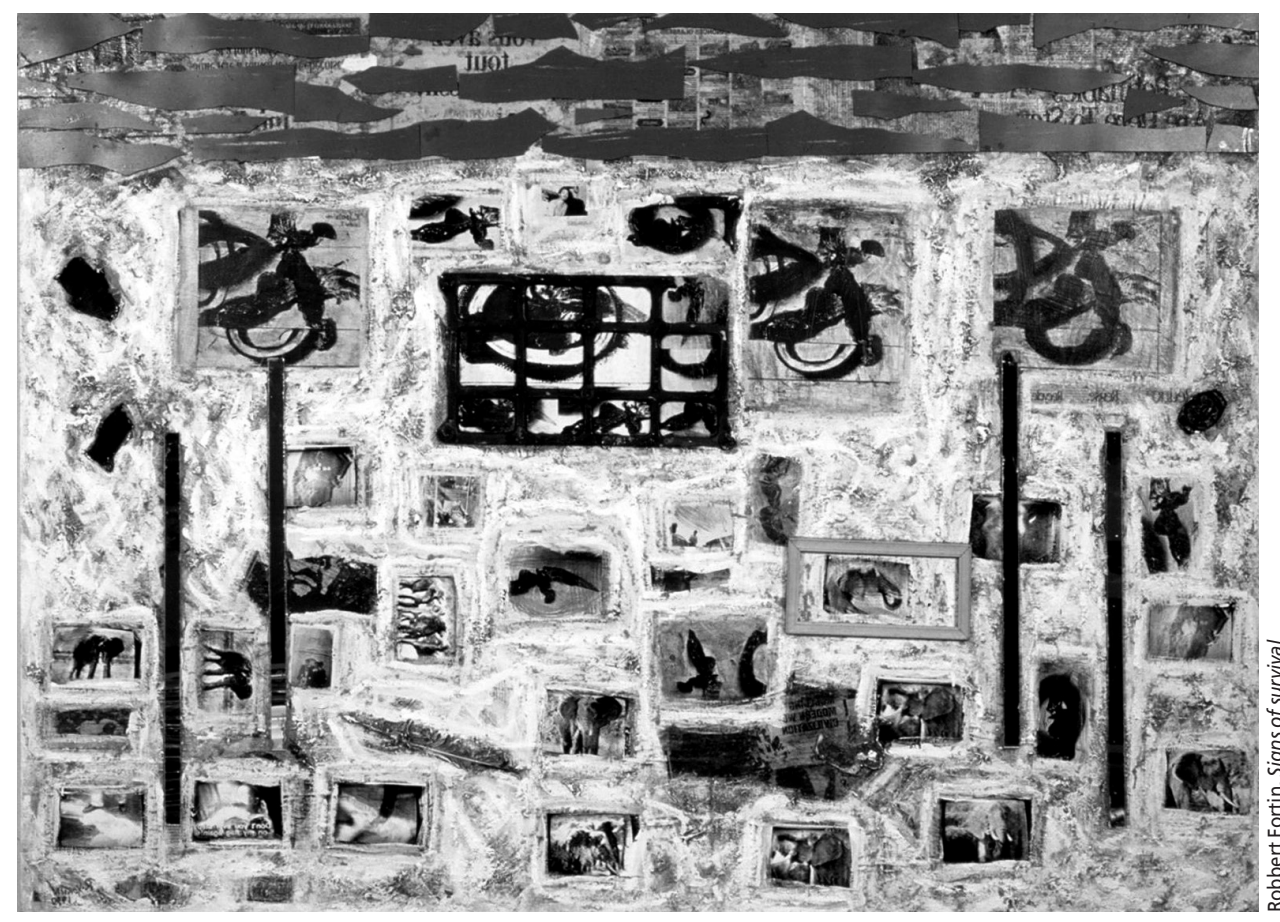

resse particulièrement aux cycles de vie. Cette "ethnologie des cycles de la vie»a connu son plein épanouissement dans les années 1960 à 1980 (principalement en Afrique au sud du Sahara). Cependant, comme le souligne, Anne-Marie Peatrick (2003), depuis une vingtaine d'années, cette anthropologie est en pleine expansion tant aux États-Unis qu'en Europe (Forner et Kertzer, 1978; Spenser, 1990; HéritierAugé, 1993). Ce qui frappe les anthropologues lorsqu'ils se penchent sur les cycles de vie, c'est l'extrême codification de la place et du statut de l'enfant. Ainsi, chez les Dogons du Mali en Afrique de l'Ouest, l'enfant occupe une place à la fois extrêmement définie et extrêmement floue. En effet, l'enfant dogon de sa conception jusqu'à son sevrage, puis du sevrage à sa puberté, s'inscrit dans sa famille d'une manière particulièrement originale. On parle d'un état larvaire, puis d'un état d'enfant (Pissani, 1983).

Il faut préciser que dans les sociétés traditionnelles africaines du Sud du Sahara, le fœtus est généralement assimilé aux animaux aquatiques (poisson, mollusque) et en particulier au silure ou poisson chat qui a la particularité de pouvoir vivre quelques temps hors de l'eau. Le fotus, être amphibien par excellence, appartient aux catégories du «mou» et de «l'humide». Mais, il est aussi considéré comme une graine, et l'enfant est assimilé à une plante en voie de maturation. Chez les Dogons, on note une forte analogie entre la croissance du mil et du sorgho et le développement de l'enfant qui est considéré comme un être riche de toutes les potentialités de la nature humaine, entièrement tourné vers l'avenir, capable d'apprendre toutes les langues, de faire sien tous les gestes et les modes de pensée (Pissani, 1983; Bibeau, 1996).

C'est précisément ce caractère d'être en devenir qui empêche le nourrisson de trouver une place, sa place dans le monde des vivants. La position de l'enfant est donc une position intermédiaire et spéculaire (c'est-àdire en miroir) à celle des morts. Mais attention pas à n'importe quels morts! Aux morts pour lesquels les rites funéraires n'ont pas encore été accomplis. Ainsi, pareillement, le défunt (le mort sans rite funéraire) et le petit enfant n'appartiennent pas au monde des vivants, mais ils n'appartiennent pas non plus à celui des morts. En fait, on peut dire que s'ils ne sont pas tout à fait morts, ils ne sont pas tout à fait vivants non plus. Au fur et à mesure de la croissance de l'enfant et des différentes étapes qui marqueront sa maturation (en premier lieu, la chute du cordon ombilical qui va signifier sa sortie définitive de l'état aquatique; puis l'apparition des premières dents, puis la marche, et le sevrage (vers 2-3 ans), l'enfant occupera cette position intermédiaire, position qu'il partage avec le défunt.

Une fois le sevrage de l'enfant fait, cette position intermédiaire qui le caractérise va perdurer d'une façon encore une fois très particulière. En effet, le nourrisson passe alors à un autre état: l'état d'enfant. Pour les Dogons, on pourrait dire que c'est à ce moment que l'enfant est considéré comme faisant partie des vivants mais là encore de vivants bien particuliers, en situation cette fois-ci spéculaire non plus à celle du défunt mais maintenant à celle du vieillard. Cette position de l'enfant, loin de lui donner un rôle négligeable (il faut considérer la place très privilégiée des personnes âgées dans les sociétés traditionnelles africaines), apporte au contraire à l'enfant un nouveau statut extrêmement original. En effet, l'enfant de cette tranche d'âge est censé percevoir directement les génies, les esprits et les sorciers. Selon les Dogons, les enfants sont les seuls, avec les initiés, à "voir dans l'eau», où ils reconnaissent par exemple le visage des ancêtres disparus. Cette position sociale en miroir à celle des vieillards affirme aussi dans le même temps la position précaire et fragilisante qu'occupe l'enfant dans son groupe, dans sa famille, dans son village. Une position extrêmement aléatoire, c'est-àdire pouvant à tout moment comme celle du vieillard d'ailleurs, basculer vers la mort. Tous les deux, l'enfant et le vieillard se situent pareillement en situation liminale pourrait-on dire avec la vie et la mort. L'un, l'enfant qui vient, qui naît, qui paraît et qui apprend à être; et l'autre, le vieillard qui suit en miroir exactement le même chemin mais à l'envers et qui lui aussi progressivement en se rapprochant de l'état de défunt apprend à disparaître, pour être ailleurs autrement.

Jusqu'à la puberté, l'enfant dogon vit avec les enfants de sa classe d'âge; il est sous le contrôle d'autres enfants plus âgés que lui, ses aînés (grands frères, grandes sœurs) mais c'est avec les enfants de sa classe d'âge qu'à la puberté il participera à un rituel initiatique bien précis qui le fera entrer dans le monde des adultes, c'est-àdire d'homme ou de femme mariable et fécondant. Cette nouvelle naissance dans le monde des adultes lui fait perdre son état intermédiaire mais, par la même occasion, il perd ses pouvoirs extraordinaires qui lui permettaient de lire dans l'eau.

Si l'on continue d'observer le cycle de vie des Dogons, on remarque que ce statut d'homme et de femme «adulte» s'achève chez l'homme lorsqu'il aura à son tour des enfants qui atteindront l'âge adulte et chez la femme à sa ménopause. Le Dogon passe alors à l'état de vieillard, et cela n'a rien de péjoratif bien au contraire (Hampaté-Ba, 1992).

À sa mort, l'individu passe à l'état de défunt, et ce, jusqu'aux funérailles finales (en règle générale, un an après le décès) qui à leur tour le font passer à l'état d'ancêtre. Puis, grâce à des rituels de fécondité effectués par les vivants, l'ancêtre pourra à son tour se réincarner lors d'une conception et revenir alors à l'état larvaire dans le monde intermédiaire afin de perpétuer et boucler ce cycle.

\section{NAISSANCE/VIE/MORT}

Avec la trilogie naissance/vie/mort, nous sommes en présence de concepts évolutionnistes centraux mais soumis à 
l'arbitraire de la culture. Le détour intentionnel que nous avons fait par l'Afrique de l'Ouest et le pays dogon nous a renseigné sur le fait que la mort et la naissance ne marquent pas obligatoirement soit la disparition du monde des vivants soit l'arrivée dans celui-ci et que les catégories d'âge qui en résultent ne sont pas des données "naturelles».

Cependant, ce n'est pas parce que de telles conceptualisations sont élaborées qu'elles prémuniront les individus qui les adoptent contre les drames et les douleurs engendrés par la mort d'un enfant. Il ne faudrait pas croire que chez les Dogons la mort d'un nourrisson ou d'un enfant ne constitue pas un drame affectif. Nul homme, nulle femme n'est à l'abri de ses émotions et l'épreuve du vide affectif est toujours une épreuve douloureuse.

Ce que l'exemple des Dogons nous invite à comprendre, c'est qu'il existe d'autres modèles explicatifs de la vie et de la mort, d'autres définitions de ces dernières, d'autres cycles de vie que les nôtres, et que les frontières de la naissance et de la mort sont soumises à l'arbitraire du culturel et non exclusivement aux lois de la biologie et de la physiologie. Mais surtout, que ces modèles explicatifs peuvent venir au bout du compte répondre en écho au chaos structurel que j'évoquais en première partie de cet article en conditionnant nos attitudes, et nos comportements «compensatoires» individuels mais aussi collectifs lors de l'apprentissage du deuil.

En d'autres termes, il s'agit ici de comprendre l'enchevêtrement de ces modèles explicatifs relatifs aux catégories d'âges et aux cycles de vie, en les percevant comme autant de réponses culturelles et philosophicoreligieuses face au profond chaos dans lequel nous plonge la mort de l'enfant. Au Mali, la mortalité périnatale est bien plus élevée qu'en Occident. Ce taux atteint des chiffres records égaux à 122\%. L'espérance de vie est en moyenne, homme et femme confondus, de 47 ans. À titre d'exemple, un million d'enfants meurent chaque année de malaria dans le monde, et les enfants du tiers monde payent un lourd tribut à ce funeste chiffre.

L'exemple des Dogons nous permet donc de comprendre qu'il peut exister différents signifiants au signifié imposé par la mort d'un enfant, et que ces signifiants sont à comprendre en fonction du macrocontexte (démographique, géographique, économique) dans lequel on vit, mais aussi en fonction des cycles de vie toujours singuliers qui se rattachent à des conceptions toujours extrêmement élaborées et structurées qui trouvent leurs origines dans le tissu culturel des groupes humains.

En Occident, par exemple, notre vision du monde et nos façons de rationaliser la vie et la mort et de nous y conformer, suivent

LES BALISES DE CE QUI EST CONSIDÉRÉ COMME VIVANT ET/OU MORT, AUPARAVANT ÉMISES ET RATIONALISÉES PAR TOUS, SONT AUJOURD'HUI EN COMPLÈTE REDÉFINITION.

une rationalité mécaniste, cartésienne, matérialiste, individualiste qui, grâce à notre biotechnologie médicale de plus en plus poussée, nous a amenés à redéfinir nos signifiants sur la mort et sur la vie en accordant une suprématie aux biosciences tout en essayant d'évacuer l'idée d'une transcendance. En effet, aujourd'hui en Occident, portés par le champ des sciences biomédicales et des sciences biotechnologiques qui les sous tendent, nous apprenons à redéfinir nos conceptions sur la vie et sur la mort. Les balises de ce qui est considéré comme vivant et/ou mort, auparavant émises et rationalisées par tous, sont aujourd'hui en complète redéfinition. À quel moment peut-on parler de vie chez un enfant? Au sein des sociétés occidentales et parfois même entre elles, le consensus scientifique, religieux, moral, éthique n'existe pas à ce sujet. La vie commence-t-elle lors de l'expulsion de l'enfant hors de la matrice maternelle ou avant? Mais à quel âge de gestation? 6 mois ? 5 mois? 4 mois et demi ou lors de la fécondation de l'ovule par le spermatozoïde paternel? L'embryon doit-il être considéré comme vivant et appartenant au monde des vivants? Les nombreux débats sur l'avortement, toujours réactivés dans nos sociétés, nous informent que nous sommes loin du consensus. L'entrée officielle dans le monde des vivants est en Occident une notion encore très incertaine et, disons-le, très peu consensuelle.

L'entrée dans le monde des morts est, elle aussi, soumise au même questionnement. En effet, la science recule à chaque jour les frontières de la mort. Aujourd'hui en Occident, nous demandons à notre biotechnologie de définir pour nous ce qu'est la vie et ce qu'est la mort. Ce qu'elle ne peut pas faire. La technologie n'est pas là pour penser ou conceptualiser. C'est aux personnes faites de chair, de sang et de parole que doit revenir ce suprême avantage. Un respirateur ne pourra jamais à lui seul prendre l'ultime décision de se "débrancher», de laisser mourir ou de laisser vivre. Et tous les jours, dans les services de réanimation de nos hôpitaux, se vivent ces moments extrêmement douloureux de décisions ultimes sur la vie et sur la mort qui se prennent en consensus à la fois avec le personnel soignant mais aussi et surtout, lorsque cela est possible, avec la famille et selon le contexte socioculturel de celle-ci.
Ce que ce détour par la logique biomédicale techniciste occidentale vient aussi nous apprendre, c'est que contrairement à ce que l'on aurait pu espérer, notre biotechnologie médicale et paramédicale ne peut nous aider à faire face au chaos engendré par la mort d'un être cher. Certes cette biotechnologie parviendra au mieux à prolonger la vie, à sauver une vie et c'est essentiel. Mais elle ne peut accomplir pour nous la quête de sens.

\section{VERS DE NOUVEAUX GLISSEMENTS SÉMANTIQUES EN OCCIDENT}

On peut observer de l'ordre biologique naissance/jeunesse/vie vers l'ordre maturité/vieillesse/vie. Ce qu'il convient aussi de remarquer, c'est que les biotechnologies médicales modernes contribuent en Occident à transformer nos conceptions sur la vieillesse et sur la jeunesse. Notre regard sur des individus que nous considérons comme vieux et sur d'autres que nous considérons comme jeunes est en train de changer. En effet, le concept de vieillesse s'est, en moins de cinquante ans, petit à petit transformé. Dans les sociétés occidentales, la longévité augmente, la natalité décroît mais dans le même temps le vieillissement s'estompe. Pour parvenir à cet état de fait, il est devenu urgent pour le bien-être de tous de rajeunir les vieux. Les exemples en chirurgie esthétique, en cosmétologie de toutes sortes, en procréation assistée, en pharmacologie ainsi que l'apparition de nouvelles spécialités biomédicales reliées à l'utilisation du laser, du botox à des fins de rajeunissement sont des exemples particulièrement éloquents sur lesquels il n'est pas nécessaire de revenir ${ }^{2}$.

Si la vieillesse s'approprie désormais la vie en niant la mort, la jeunesse quant à elle se retrouvera spontanément connectée à la mort. Ainsi, lorsque de façon consensuelle une société décide d'inverser le rapport classique Vieillesse/Mort par Vieillesse/ Vie, on comprend qu'elle puisse avoir des difficultés à rationaliser culturellement cet autre glissement sémantique qui s'effectuera entre désormais Jeunesse et Mort. On se doute que les transformations socioculturelles qui en découleront risquent, dans un premier temps, de nous apparaître paradoxales. Ces paradoxes prennent pourtant la forme de constats. Il semble, en effet, que plus la longévité augmente, plus 
les vieux en s'imposant par leur nombre veulent rajeunir et plus la jeunesse en tant que telle et avec elle les enfants tendent tranquillement à disparaître physiquement de nos sociétés occidentales. On parle de dénatalité parfois alarmante dans certains pays occidentaux (le Québec est d'ailleurs un exemple éloquent à ce sujet). On évoque aussi un malaise des sociétés occidentales (et le mot est faible) par rapport aux structures familiales qui se transforment, aux familles qui diminuent et au nombre de jeunes (enfants et adolescents) qui décroît. De plus, les jeunes composent un groupe d'âge à risque: à risque de violence, de délinquance, de perturbation de l'ordre public. Bref, en fonction de leur nombre de moins en moins élevé, ils dérangent plus qu'ils n'encombrent. Çà et là, on voit même parfois émerger de véritables réactions d'exclusion à leur égard. Ainsi certains endroits publics leur sont parfois interdits. On parle d'hôtels, de restaurants, de cinémas, de théâtres (même accompagnés par des adultes), voire les rues dans une certaine municipalité du Québec qui leur sont interdites le soir... ${ }^{3}$. Si Jeunesse et Mort ne se conceptualisent pas ensemble "émotivement» (nous l'avons illustré dans la première partie de cet article), ils se conjuguent pourtant matériellement, objectivement dans notre vie quotidienne.

Comme nous venons de l'illustrer en Occident, avec la disparition, l'effacement progressif des jeunes dans nos sociétés (dénatalité, réactions d'exclusion visant les jeunes), il est normal que Vieillesse et Vie se retrouvent du coup immanquablement associées. En fait, cela nous permet de réaffirmer qu'il est impossible de définir des catégories d'âges sans comprendre auparavant les bases conceptuelles sur lesquelles ces catégories reposent. Ces bases se composent d'un enchevêtrement de considérations philosophico-religieuses portant principalement sur les catégories Vivant/Mort (ou plutôt sur ce que l'on considère comme étant vivant et sur ce que l'on considère comme étant mort). En Occident, les deux catégories d'âge Vieillesse et Jeunesse sont elles-mêmes à comprendre en fonction des liens étroits qui les unissent avec leurs catégories de base qui sont Naissance/Mort. Ces catégories se définissent rétroactivement les unes par rapport aux autres et doivent toujours être comprises ensemble. Nous entrons là dans une réelle circularité phénoménologique. De plus, ces catégories conceptuelles sont en équilibre instable, pourrait-on dire, les unes avec les autres, car elles interagissent constamment en venant nous questionner et en interpellant notre quête de sens.

Corrélativement à ces nouvelles réalités sémiotiques liées à une technicisation de nos vies et de nos morts associée à un scientisme de plus en plus poussé, nous voyons en Occident émerger ce qui semblerait faire office de nouveaux cadres philosophicoreligieux venant, dans les faits, essayer de réglementer en rationalisant ces nouvelles réalités sémantiques relatives à la vie, à la mort, à la jeunesse et à la vieillesse. Çà et là en Occident, et variant selon les pays concernés, de nouvelles «réglementations » voient le jour. Ces nouvelles réglementations tentent toutes à l'intérieur d'un cadre strictement laïque, de légiférer, d'encadrer toutes recherches et toutes pratiques biomédicales portant sur des êtres humains. Ce sont désormais à travers des grilles légales, juridiques et éthiques que nous appréhendons les concepts de vie et de mort. On parle désormais de bioéthique. Il est, en effet, devenu urgent d'encadrer éthiquement les sciences biomédicales. Est-il légitime qu'à 65 ans on puisse de nouveau enfanter ${ }^{4}$ ? Un embryon à quatre méioses, conservé dans une pipette en chambre froide à moins $80^{\circ} \mathrm{C}$, est-il un être à part entière déjà né et donc vivant? La mort se définitelle désormais lors de l'arrêt des fonctions vitales non techniquement assistées? Mais même encore là, faut-il se faire "cryogéniser» après notre mort tant la définition de la mort elle-même est soumise dans nos imaginaires à l'aléatoire de notre technologie et au temps? En Occident, nos définitions sur les concepts de jeunesse, de vieillesse, de vie et de mort se complexifient énormément et sont tout comme notre monde social et culturel, en complète redéfinition.

Nos nouvelles taxonomies biomédicales viennent rendre compte sans contredit de notre vision du monde actuel, de notre quotidien, de nos technicités et de notre matérialisme scientifique. Ces nouvelles taxonomies ont une emprise sur nos comportements individuels et collectifs face au phénomène de la naissance et de la mort, et, disons-le, de la vie elle-même. Se distanciant pour des raisons de scientificité du cadre philosophico-religieux dans lesquelles elles étaient ancrées depuis des siècles, ces nouvelles conceptualisations de la naissance, de la mort et avec elles de la jeunesse et de la vieillesse semblent désormais être encadrées par des principes d'éthique et de bioéthique (principes de bienfaisance et de non-malfaisance essentiellement) qui tentent d'adapter nos pratiques médicales à une conformité morale et éthique en évacuant tout caractère de transcendance pouvant être relié à ces réalités.

Comme nous l'avons vu précédemment, les Dogons ont élaboré un cycle de vie extrêmement sophistiqué, où le nourrisson et le défunt, l'enfant et le vieillard s'agencent deux à deux de façon extrêmement originale. En Occident, de notre côté, devant nos nouvelles incertitudes, nous sommes en train d'élaborer des catégories générationnelles et de nouveaux cycles de vie tout aussi sophistiqués et complexes. Ces nouvelles catégorisations tentent désormais de définir les rapports originaux qu'entretiennent Vieillesse et Vie ainsi que de nouveaux rapports pour le moins inédits entre Vieillesse et Naissance, et Naissance et Mort. Les glissements sémantiques de ces catégorisations ont permis aux scientifiques occidentaux de redécouvrir ce que les médecins de l'Inde antique ${ }^{5}$ (1999) avaient déjà pressentis quelque 1500 ans avant J.-C., à savoir que les phénomènes de naissance et de mort sont à comprendre dans le même temps car, dans les faits, ils présentent de nombreuses analogies ${ }^{6}$.

Le détour effectué en Afrique de l'Ouest nous a permis de comprendre que les taxonomies dogons établissent des liens spéculaires entre les catégories d'âge suivantes: Naissance/Défunt et Enfant/Vieillard. En effet, le nouveau-né dogon est en position spéculaire au regard du défunt et le jeune enfant, en position spéculaire au regard $\mathrm{du}$ vieillard. A priori, il semblerait donc que les liens existants entre ces différentes catégories conceptuelles ne soient pas si éloignés de ceux existant dans les nouvelles conceptualisations occidentales à ce propos et décrites précédemment. Pourtant, il n'en est rien. Leurs bases sémantiques et paradigmatiques sont très éloignées les unes des autres. La version dogon repose sur une cosmologie mythologique extrêmement élaborée (Bibeau, 1996) et la version occidentale repose sur une scientificité techniciste et laïque elle aussi extrêmement élaborée.

Dans nos pratiques médicales occidentales de type pédiatrique, avec une clientèle de plus en plus pluriculturelle et multiethnique, ces conceptualisations sur la vie, la mort, la jeunesse et la vieillesse a priori

CONTRAIREMENT À CE QUE L'ON AURAIT PU ESPÉRER, NOTRE BIOTECHNOLOGIE MÉDICALE ET PARAMÉDICALE NE PEUT NOUS AIDER À FAIRE FACE AU CHAOS ENGENDRÉ PAR LA MORT D'UN ÊTRE CHER. 
similaires et pourtant si différentes l'une de l'autre sont parfois difficiles à rationaliser de part et d'autre. Surtout, pourrait-on dire, lorsque le personnel soignant occidental et une famille dogon, par exemple, se retrouvent plongés au cœur du chaos que la perte d'un enfant immanquablement provoque. Ainsi, lorsque le personnel soignant d'un hôpital moderne occidental, et avec lui la société occidentale tout entière, se trouve confronté à d'autres conceptualisations relatives à la mort et à la naissance (et ce, même si les liens sémantiques entre les catégories sont assez similaires), ces «autres» catégories peuvent être perçues par ce même personnel médical et paramédical comme des conceptualisations passéistes, car empruntes et issues de convictions mystico-philosophico-religieuses qui, dans un monde cartésien, laïque, scientifique et matérialiste occidental ont de moins en moins leurs raisons d'être car ayant perdu toute crédibilité. Ces «autres» convictions s'actualiseront dans des comportements, des attitudes et des réactions de deuil et d'acceptation ou de refus de celui-ci. Il en résulte que les familles endeuillées pourront alors difficilement se faire comprendre, voire se faire entendre par les professionnels de santé occidentaux. Il faut savoir que ces malentendus et ces non-entendus sont réciproques. Ils proviennent d'un côté d'une technicisation parfois outrancière de la naissance et de la mort; et de l'autre d'un attachement persistant à des convictions mystico-philosophico-religieuses reliées à ces mêmes concepts. Pourtant, comme je l'ai démontré dans mon propos, la logique structurelle de l'agencement des catégories relatives à la naissance, à la mort et avec elles à la jeunesse et à la vieillesse ainsi que leur agencement particulier en des paradigmes Naissance/Jeunesse/Mort et Maturité/Vieillesse/Vie demeurent de part et d'autre, autant chez les Dogons traditionalistes et religieux que chez les Occidentaux technicistes et laïques d'aujourd'hui, structurellement assez équivalents. Ainsi, en tentant de faire ressortir l'incroyable plasticité des croyances et des pratiques qui se mettent en place particulièrement autour de la mort de l'enfant, il est intéressant de remarquer qu'en ce début de $\mathrm{XXI}^{\mathrm{e}}$ siècle en Occident nous nous retrouvions devant des catégories conceptuelles paradigmatiques quasi identiques à celles des Dogons: liant Vieillesse et Vie ainsi que Jeunesse et Mort même, et surtout, si elles véhiculent des sens parfois diamétralement opposés.

\section{Bibliographie}

ASTANGA-HRDAYA OFVAGBHATA(1999). THE BOOK OF EIGHT BRANCHES OF AYURVEDA, translated by A Board of Scholars, 3 vols., 1263 p.

BIBEAU, G. (1996). Les errances du Renard pâle dans le haut pays dogon, Culture XVII (1-2), p. 61-80.

BIBEAU, G et C. CHARLAND (1994). "Sur la piste des errances du Renard pâle. Les recherches d'une équipe italo-malienne sur le haut-plateau dogon», Psychopathologie africaine, vol. XXVI, $\mathrm{n}^{\circ} 3$, p. 341-385.

BOURDIEU, P. (1984). La «jeunesse» n'est qu'un mot, Questions de sociologie, Paris, Éd. De Minuit, p. 143-154.

DES AULNIERS, L. (1996). Itinérances de la maladie grave. Le temps des nomades, Paris, L'Harmattan.

DOUGLAS, M. (1968). «Dogon Culture: Profane and Arcane», Africa, 38, p. 16-24.

FIORE, B. (1988). Anthropologie de la petite enfance et de l'éducation dans les pays africains, dans Piero Copro (éd.), p. 164-188.

FIORE, B. et D. TOGO (1990). Notes ethnologiques de la naissance chez les Dogons, dans Piero Copro et Arouna Keita (éd.), p. 309-320.

FORNER, F. et D KERTZER (1978). «Transitions over Life Course: Lessons from Age-sets Societies ", American Journal of Sociology, vol. 83, n 5, p. 1081-1104.

GEBRE-EGZIABHER, S. (1993-1994). Africans' Contributions to Science: A Culture of Excellence. Equity Coalition, University of Michigan School of Education, vol. 3, n ${ }^{\circ}$ 2, p. 30-31.

HAMPATÉ-BÂ, A. (1992). Amkoullel, l'enfant peul, Actes Sud.

HÉRITIER-AUGÉ, F. (1991-1993). Les âges de la vie, Séminaire du Collège de France.

LÉVI-STRAUSS, C. (1977), Anthropologie Structurale, Paris, Plon.

PEATRICK, A.-M. (2003). L'océan des âges, L'Homme, p. 167-168.

PISSANI, L. (1983). Enfants Dogon, Vidéo, 42 min., Rome, C.N.R. Instituto Di Psicologia.

RABIN, J. (1994). L'enfant du lignage. Du sevrage à la classe d'âge, Paris, Payot.

RABIN-JANIN, J. (2003). Enfance, âge et développement chez les Wolof du Sénégal, L'Homme.

RAIMBAULT, G. (1996). Lorsque l'enfant disparaît, Paris, Odile Jacob.

RIESMAN, P. (1988-1989). «La personne et le cycle de vie dans la vie sociale et la pensée africaines ", Psychopathologie africaine, vol. 22, no 3, p. 327-363

SPENSER, P. (dir.) (1990). Anthropology and the Riddle of Sphinx. Paradoxes of Change in the Life Course, Londres, Routledge (ASA Monographs, 28).
THOMAS, L.-V. (2000). La mort en question. Traces de mort, mort des traces, Nouvelles études anthropologiques, Paris, L'Harmattan.

THOMAS, L.-V. (2003). La mort, Presses universitaires de France.

VIDAL, J.-M. (2000). "Perspectives transculturelles sur la mort et le deuil », Revue du Québec sur le cancer du sein (REIQCS), Spécial Soins palliatifs, vol. 6, nos 1-2, p. 62-67.

STATISTIQUES, Le Mali en bref (2001) <http:// erpe.saliege.com/le_Mali_en_bref.htm.>

\section{Notes}

1. Le docteur Jean-Michel Vidal a étudié la philosophie, la médecine, l'ethnologie et la médecine tropicale à Montpellier en France. Arrivé à Montréal, ses études se sont poursuivies à l'Université de Montréal (doctorat en anthropologie socioculturelle sous la direction du professeur G. Bibeau) et à l'Université McGill (postdoctorat sous la direction du professeur E. Corin). Le docteur Vidal est actuellement professeur associé et il enseigne l'anthropologie médicale dans différentes facultés à l'Université de Montréal (Sciences biomédicales, Faculté de médecine dentaire, Faculté d'éducation permanente, Département d'anthropologie) et il est directeur de projets cliniques pour la compagnie Genizon BioSciences.

2. Ces nouvelles techniques biomédicales esthétiques visent aussi à redonner une image du corps et par là même de soi, conforme aux canons esthétiques en vigueur. À ce titre, ces techniques se pratiquent aussi sur des individus jeunes.

3. Voir les récents évènements au Québec (été 2004) relatifs au désir de législation du maire de la municipalité de Huntington d'imposer aux jeunes de sa ville un couvre-feu le soir à $10 \mathrm{~h} 30$.

4. Voir l'expérience médicale récente menée en Italie sur une femme de 65 ans (donc ménopausée) qui a pu, grâce à une hormonothérapie appropriée, être inséminée artificiellement et mener une grossesse à terme. On remarquera dans cet exemple que le lien sémantique liant vieillesse et vie est illustré dans cette expérimentation médicale de façon presque caricaturale.

5. En effet, le traité de médecine ayurvédique le Vagbhata dit aussi le «Corpus de l'essence de l'Ayurveda en huit articles» consacre l'entièreté du Livre du Corps à traiter des premiers signes de la vie et des signes de la mort. Charaka, médecin ayurvédique de l'Inde antique, a établi des taxonomies médicales particulières dans lesquelles il se propose d'étudier les phénomènes de la vie (de naissance) et de la mort dans le même temps. Les premiers signes de la vie et les signes avantcoureurs de la mort doivent, selon lui, être compris ensemble et dans le même temps.

6. Mitose, méiose, apoptose et nécrose cellulaire sont pour les biologistes moléculaires des phénomènes biologiques en équilibre instable les uns les autres et à comprendre ensemble dans l'étroite relation qu'ils entretiennent avec le système immunitaire. Ces phénomènes sont à l'origine de la genèse, de la croissance, de la mort cellulaire et donc de la vie et de la mort. 